\title{
Study on the Deformation of Pre-Stressed Centre Diaphragm of Pressure Sensor
}

\author{
Shenzhi He ${ }^{1}$, Guoqing $\mathrm{Hu}^{1}$, Yonghong Zhou ${ }^{2}$, Jianling Wang ${ }^{2}$, Chong Zou ${ }^{2}$, Jahangir Alam SM ${ }^{*}$ \\ ${ }^{1}$ School of Mechanical \& Automotive Engineering, South China University of Technology, Guangzhou 510640, China; \\ ${ }^{2}$ Fujian Wide Plus Precision Instruments CO., LTD, Fuzhou 350015, China \\ *Corresponding authors E-mail: mejahangir@scut.edu.cn
}

\begin{abstract}
The centre diaphragm is a key part of the silicon piezoresistive pressure sensor with three diaphragms structure which is one major matter to the sensitivity of the output pressure value. The characteristics between the deflection at the center of the circular diaphragm and the pressure loaded on it are considered by applying the energy theory. And the nonlinear deflection and the maximal deflection formulas are derived by taking the outside pressure loaded and the prestress into account. The simulation of the large deflection of the diaphragm is developed by the ANSYS finite-elementmethod (FEM) software. And the pre-stress is loaded by applying temperature loads in the APDL module. Additionally, the maximal deflection of the centre diaphragm is measured by the micro displacement test equipment. And the testing results confirm both the correction of mathematical model and the validity of the ANSYS FEM simulation software. The results indicated that the pre-stress affect the deflection of the diaphragm of pressure sensor obviously and should not be ignored.
\end{abstract}

Keywords-silicon pressure sensor; centre diaphragm; deflection; pre-stress; energy; temperature load.

\section{INTRODUCTION}

The high accuracy silicon pressure sensor (SPS) which can bear the extreme high temperature and high pressure environment is a hot-spot of research [1]. In order to enhance the anti-overload capacity during the design of pressure sensor, a structure of three diaphragms, one centre diaphragm and two isolation diaphragms is adopted. And the three diaphragms structure is shown in Fig. 1[2].

The perturbation method is often used to establish an approximate numerical analysis of the equations of the deflection, especially dealing with the complicated nonlinear equilibrium equations [3]. Chien W Z [4] proposed the perturbation method which set the deflection at the center of the diaphragm as the perturbation parameter. ZHOU M X [5], et al. obtained the analytical equations of the deformation and stress distribution of rectangular film by applying the energy theory. This paper is contained the approximate numerical solution of the deflection of circle diaphragm of pressure on load by applying the minimum energy theory, and the pre-stress is included. In Fig. 1, the numerical number are shown to describe the model as, 1isolation diaphragm of high pressure sector, 2-isolation diaphragm of low pressure sector, 3-centre diaphragm, 4 -oil tunnel, 5- sensitive sensor, 6-ceramic plate, 7-

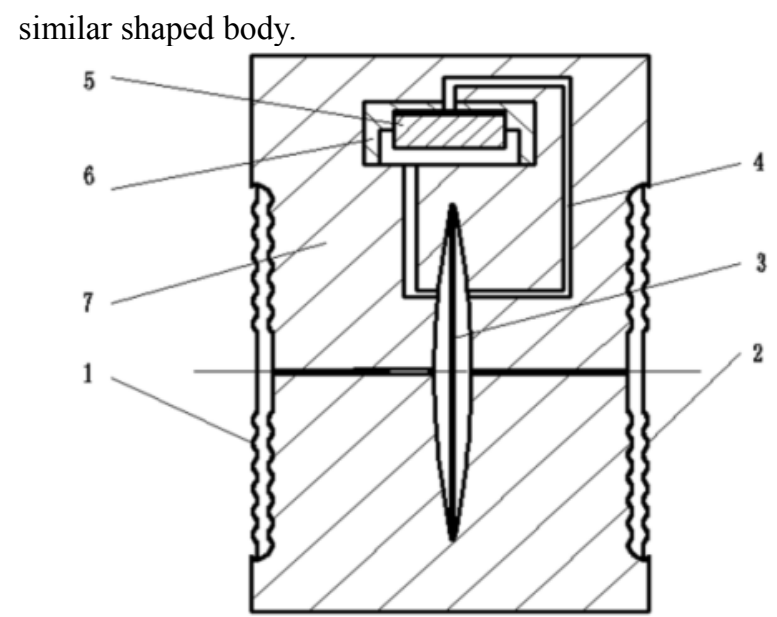

Figure 1. Diaphragm-box overload-resistance structure model

\section{DEFLECTION THEORY OF CIRCLE DIAPHRAGM}

It is considered in here a common simple structure of thin circle diaphragm as shown schematically in Fig. 2.The radius from the centre axis is expressed by $r$, another direction is denoted by $t$ which is tangent to the circumference, the diaphragm radius is $a$, and the thickness is $h$. The whole elastic potential energy of diaphragm, $V$, can be divided into three parts: the bending energy in response to the deflection of the diaphragm is $V_{l}$, the deformation energy generated by the deformation of the neutral plate is $V_{2}$, and the residual strain energy resulted by the weld pre-stress of diaphragm is $V_{3}$.

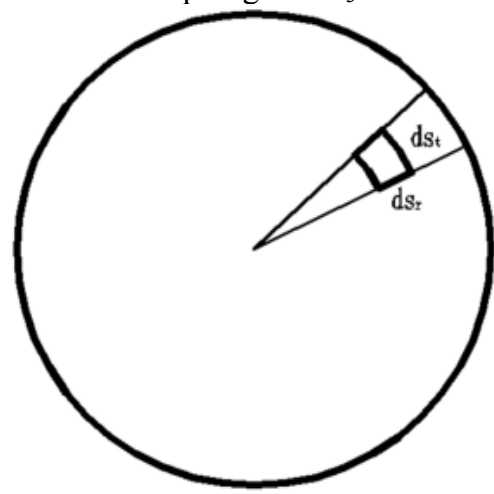

Figure 2. Coordinate simplified model 
The bending energy $V_{l}$ is expressed as [6],

$$
V_{1}=\frac{32 \pi}{3} \frac{\omega_{0}^{2}}{a^{2}} D
$$

where $D$ is the bending rigidity,

$$
D=\frac{E h^{3}}{12\left(1-\mu^{2}\right)}
$$

where, $E, \mu$ and $\omega_{0}$ are Young's modulus, Poisson's ratio and the maximal deflection of the diaphragm, respectively. The deformation energy of the neutral plate $V_{2}$ is expressed as [6]:

$$
V_{2}=\frac{\pi E h}{1-\mu^{2}} \int_{0}^{a}\left(\varepsilon_{r}^{2}+\varepsilon_{t}^{2}+2 \mu \varepsilon_{r} \varepsilon_{t}\right) \cdot r d r
$$

where $\varepsilon_{r}$ and $\varepsilon_{t}$ are the strain of the direction of $\mathrm{r}$ and the direction of t, respectively. $N_{r}$ and $N_{t}$ are the stress of the direction of $\mathrm{r}$ and the direction of $\mathrm{t}$, respectively. Assuming the residual stress in the neutral plate is $\sigma$ (stress of tension), so the residual stress energy $V_{3}$ is expressed as [6]:

$$
V_{3}=2 \pi \int_{0}^{a} \frac{\sigma \varepsilon_{r}}{2} r d r
$$

That is to say, the formula of the whole potential energy $V$ is written as,

$$
V=V_{1}+V_{2}+V_{3}
$$

If the applied uniform pressure which is in the norm of the plate is $P$, the work in response to the uniform pressure $P$ is expressed as:

$$
W=\iint(P \cdot w) d S=\int_{0}^{2 \pi} \int_{0}^{a} P \cdot w \cdot r d r d \theta
$$

According to the minimum energy theory, the work of loaded pressure completely convent into the total potential energy at the balance state, namely:

$$
V=W
$$

Consider the case of pre-stress exiting ( $V_{3}$ could not be ignored). Here, it is assumed Poisson's ratio, $\mu$, equal to 0.3 . And the shape function of the deformation of the diaphragm can be expressed approximately as:

$$
u=r(a-r)\left(C_{1}+C_{2} r\right)
$$

where we get the simplified function of $u$ refer to $r$ by ignoring the effect of high order component. The weld prestress of diaphragm is the stress of tension, and the residual stress energy $V_{3}$ is expressed as,

$$
V_{3}=2 \pi \int_{0}^{a} \frac{\sigma \varepsilon_{r}}{2} r d r=\pi \int_{0}^{a} \sigma\left[\frac{d u}{d r}+\frac{1}{2}\left(\frac{d \omega}{d r}\right)^{2}\right] r d r
$$

We can get the unknown constants, $C_{l}$ and $C_{2}$, by the minimum energy theory:

$$
\left\{\begin{array}{l}
\frac{\partial V}{\partial C_{1}}=\frac{\partial\left(V_{1}+V_{2}+V_{3}\right)}{\partial C_{1}}=0 \\
\frac{\partial V}{\partial C_{2}}=\frac{\partial\left(V_{1}+V_{2}+V_{3}\right)}{\partial C_{2}}=0
\end{array}\right.
$$

Then, we obtain the total potential energy:

$$
V=\frac{32}{3} \pi D\left[\frac{\omega_{0}{ }^{2}}{a^{2}}+0.243 \cdot \frac{\omega_{0}{ }^{4}}{a^{2} h^{2}}-0.1850 m \frac{\omega_{0}{ }^{2}}{h^{2}}-0.780 m^{2} \frac{a^{2}}{h^{2}}\right]
$$

where $m$ is the coefficient of the residual stress, and $m$ is a negative factor. And the loaded pressure of the diaphragm can be described as:

$$
P=\frac{64 D}{a^{4}}\left[\omega_{0}+0.486 \frac{\omega_{0}{ }^{3}}{h^{2}}+0.185|m| \frac{a^{2} \omega_{0}}{h^{2}}\right]
$$

\section{RELATION BETWEEN MAXIMAL DEFLECTION AND APPLIED PRESSURE}

In order to obtain the relation character between the maximal deflection and the applied pressure, the centre diaphragm which is in the case of applied uniform pressure is analyzed by using the ANSYS APDL module.

The pre-stress of diaphragm is the residual stress of tension which is resulted by the cooling of weld diaphragm. So it is effective to load the pre-stress by the function of thermal expansion of material. And it will be simulated by applying several temperature loads of different parts.

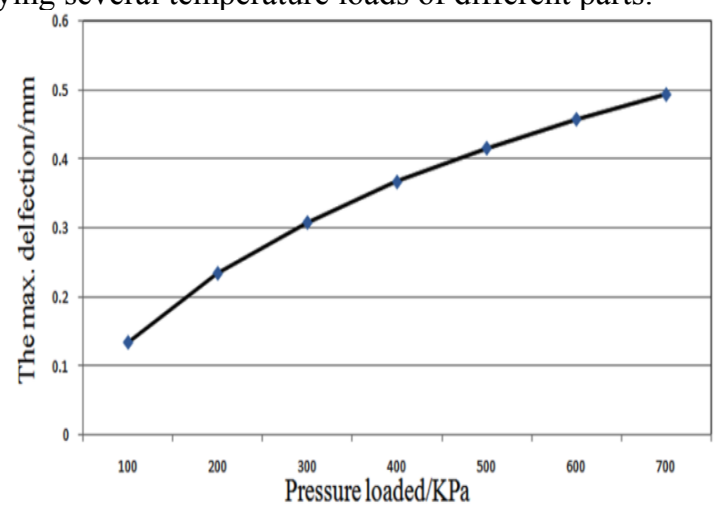

Figure 3. Simulation of maximal deflection-pressure relationship

In the Fig. 3, the relation character between the maximal deflection and the applied pressure is described. It can be obtained from Fig. 3 that it is of obvious non-linearity character in the case of large deflection.

\section{RESUlT AND DISCUSSION}

Applying the micro displacement measurement equipment, the relation characteristics between the maximal deflection and the applied pressure of pressure sensor with span of $40 \mathrm{Kpa}$ is obtained. The error of simulation value which is contrasted to the actual test value is expressed in the Fig. 4. It is obtained from the Fig. 4 that the error of simulation value is less than $3 \%$ in the pressure span of $(250 \mathrm{KPa}, 700 \mathrm{KPa})$ where large deflection happened, while the error is much larger in the span of $(100 \mathrm{KPa}, 250 \mathrm{KPa})$. This can be explained by the factors as follow: Firstly, there is theoretical error of large deflection model of simulation in the case of the transition stage from the small deflection to the large deflection when the maximal deflection $\lambda$ within the span of $[0.3 \mathrm{~h}, \mathrm{~h}]$. Secondly, the actual measured value is of larger relative error if the displacement of diaphragm is small. 


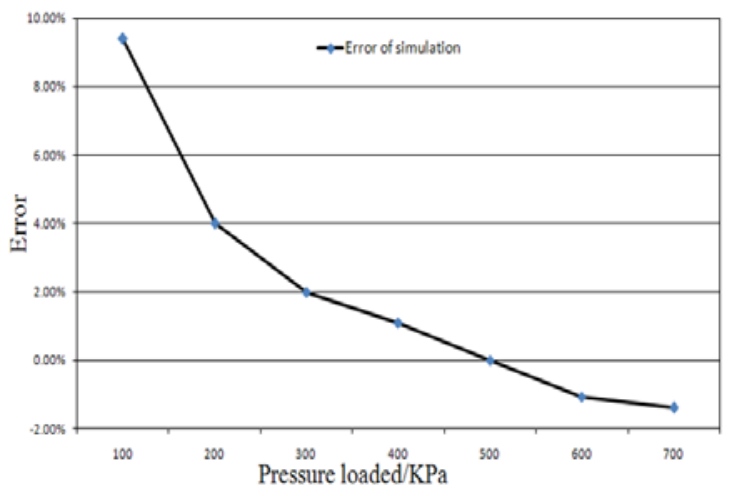

Figure 4. ANSYS simulation model error curve

The error of the theoretical value of maximal deflection which is contrasted to the actual test value is described in the Fig. 5.

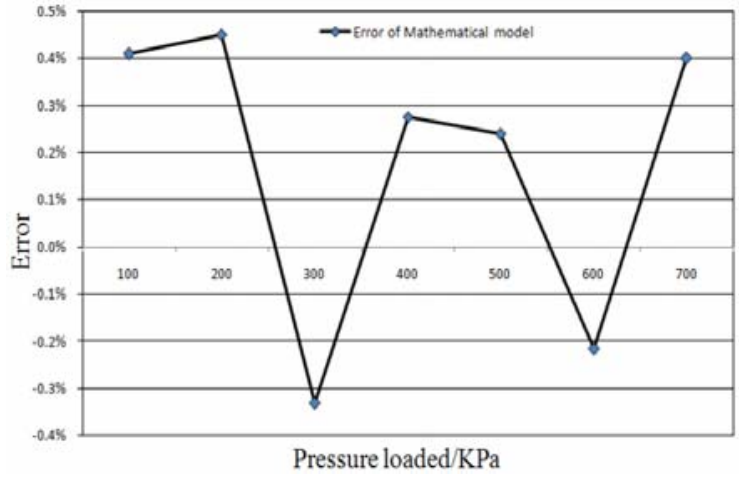

Figure 5. Mathematical model error curve

It is obtained from the Fig. 5 that the maximal error of theoretical value is less than $0.5 \%$ and the error of theoretical value is of variability crossing the zero line. As the Eq.(11) can include the case of small deflection, the error of theoretical value is less than the error of simulation value.

\section{CONCLUSIONS}

This research paper demonstrates the deflection theory of the centre diaphragm of the pressure on load and prestress is included. And the deflection character is simulated by ANSYS software. Finally, the testing results of the actual value of deflection confirm the high accuracy both the theoretical value and the simulation value. It is essentially beneficial to the design of the diaphragm and the whole sensor.

\section{ACKNOWLEDGEMENTS}

The research work is supported by Chinese 863 projects, No. 2014AA042000.

\section{REFERENCES}

[1] Suzuki K., Ishihara T. \& Hirata M., Nonlinear analysis of a CMOS integrated silicon pressure sensor. IEEE Transactions on Electron Devices, 34(6), pp. 1360-1367, 1987.

[2] Wang S.J., Overload Protection Design of Silicon Pressure Sensor. Instrument technique and sensor, 35(1), pp. 7-8, 2010.

[3] Adams, G.G., Elastic wrinkling of a tensioned circular plate using von Karman plate theory. Applied Mechanics, Transactions ASME, 60(2), pp. 520-525, 1993.

[4] Chien, W.Z., Huang Q. \& Feng W., Three dimensional stress analysis of symmetric composite laminates under axial extension and in-plane pure shear. Applied Mathematics and Mechanics, 15(2), pp. 101-108, 1994.

[5] Zhou, M.X, Huang, Q.A., \& Qin M., Modelling, design \& Fabrication of a triple-layered capacitive pressure sensor. Sensors \& Actuators, A(117), pp. 71-81, 2005.

[6] Timoshenko, S.P. \& Krieger, S.W., Theory of Plates and Shells. 20th edition, New York: McGraw-Hill, 1970. 